\title{
The multi-biomarker disease activity score tracks response to rituximab treatment in rheumatoid arthritis patients: a post hoc analysis of three cohort studies
}

Nadia M. T. Roodenrijs ${ }^{1 *}$, Maria J. H. de Hair ${ }^{1}$, Gill Wheater ${ }^{2}$, Mohsen Elshahaly ${ }^{3}$, Janneke Tekstra ${ }^{1}$, Y. K. Onno Teng ${ }^{4}$, Floris P. J. G. Lafeber ${ }^{1}$, Ching Chang Hwang ${ }^{5}$, Xinyu Liu ${ }^{5}$, Eric H. Sasso ${ }^{5}$ and Jacob M. van Laar ${ }^{1}$

\begin{abstract}
Background: A multi-biomarker disease activity (MBDA) score has been validated as an objective measure of disease activity in rheumatoid arthritis (RA) and shown to track response to treatment with several disease-modifying antirheumatic drugs (DMARDs). The objective of this study was to evaluate the ability of the MBDA score to track response to treatment with rituximab.

Methods: Data were used from 57 RA patients from three cohorts treated with rituximab $1000 \mathrm{mg}$ and methylprednisolone $100 \mathrm{mg}$ at days 1 and 15. The MBDA score was assessed in serum samples obtained at baseline and 6 months. Spearman's rank correlation coefficients were calculated for baseline values, 6 -month values, and change from baseline to 6 months $(\triangle)$, between MBDA score and the following measures: disease activity score assessing 28 joints (DAS28) using erythrocyte sedimentation rate (ESR) or high-sensitivity C-reactive protein (hsCRP), ESR, (hs)CRP, swollen and tender joint counts assessing 28 joints (SJC28, TJC28), patient visual analogue scale for general health (VAS-GH), health assessment questionnaire (HAQ), and radiographic progression over 12 months using Sharp/van der Heijde score (SHS), as well as six bone turnover markers. Additionally, multivariable linear regression analyses were performed using these measures as dependent variable and the MBDA score as independent variable, with adjustment for relevant confounders. The association between $\triangle M B D A$ score and European League Against Rheumatism (EULAR) response at 6 months was assessed with adjustment for relevant confounders.
\end{abstract}

Results: At baseline, the median MBDA score and DAS28-ESR were 54.0 (IQR 44.3-70.0) and 6.3 (IQR 5.4-7.1), respectively. MBDA scores correlated significantly with DAS28-ESR, DAS28-hsCRP, ESR and (hs)CRP at baseline and 6 months. $\triangle M B D A$ score correlated significantly with changes in these measures. $\triangle M B D A$ score was associated with EULAR good or moderate response (adjusted $\mathrm{OR}=0.89,95 \% \mathrm{Cl}=0.81-0.98, p=0.02$ ). Neither baseline MBDA score nor $\triangle \mathrm{MBDA}$ score correlated statistically significantly with $\triangle \mathrm{SHS}(n=11)$ or change in bone turnover markers $(n=23)$, although $\triangle \mathrm{SHS} \geq 5$ was observed in 5 (56\%) of nine patients with high MBDA scores.

Conclusions: We have shown, for the first time, that the MBDA score tracked disease activity in RA patients treated with rituximab and that change in MBDA score reflected the degree of treatment response.

Keywords: Rheumatoid arthritis, Disease activity, Treatment response, MBDA score, Biomarkers, Rituximab

\footnotetext{
*Correspondence: n.m.t.roodenrijs@umcutrecht.nl

'Department of Rheumatology \& Clinical Immunology, University Medical

Center Utrecht, Heidelberglaan 100, 3508 GA Utrecht, The Netherlands

Full list of author information is available at the end of the article
}

(c) The Author(s). 2018 Open Access This article is distributed under the terms of the Creative Commons Attribution 4.0 International License (http://creativecommons.org/licenses/by/4.0/), which permits unrestricted use, distribution, and reproduction in any medium, provided you give appropriate credit to the original author(s) and the source, provide a link to the Creative Commons license, and indicate if changes were made. The Creative Commons Public Domain Dedication waiver (http://creativecommons.org/publicdomain/zero/1.0/) applies to the data made available in this article, unless otherwise stated. 


\section{Background}

Rheumatoid arthritis (RA) is the most common, chronic inflammatory joint disease, characterised by synovitis, joint damage, and systemic immune and inflammatory manifestations. Achieving remission or low disease activity is the main treatment goal in order to prevent joint damage and disability [1]. The European League Against Rheumatism (EULAR) and the American College of Rheumatology (ACR) recommend regular assessment of the level of disease activity $[2,3]$. The disease activity score assessing 28 joints (DAS28) is one of the most frequently used composite scores for the assessment of disease activity in clinical studies of RA [4]. However, the DAS28 has shortcomings that hamper its use in clinical practice [5]. It does not include the ankles or feet, whereas these are common sites of inflammation in RA. Moreover, the DAS28 contains subjective components, making it highly variable between and within assessors and unreliable at the patient level. In addition, RA inflammation can be extra-articular, which is not readily detected by the DAS28. Thus, there is a need for an objective measure that reflects systemic disease activity and is sensitive to change. It would be of additional benefit if that measure could be used to predict radiographic progression.

The multi-biomarker disease activity (MBDA) score is based on biochemical markers only. It is thus more objective than the DAS28 and may potentially be a better indicator of systemic inflammation. The MBDA score is calculated with an algorithm that uses the concentrations of 12 serum protein biomarkers to produce a score, on a scale of 1 to 100, that represents the level of disease activity in patients with RA [6]. The MBDA score has been validated based on its correlation with DAS28 using C-reactive protein (CRP) and other clinical disease activity measures $[7,8]$. The clinical validation of the MBDA score is supported by evidence that the MBDA score is a stronger predictor of radiographic progression than DAS28-CRP, and that it predicts radiographic progression when it is discordant with DAS28-CRP (e.g. when DAS28 is low and MBDA score is high) [9].

The MBDA score tracks response to a variety of disease-modifying anti-rheumatic drugs (DMARDs), including methotrexate [10] (with and without prednisone [11]), tumour necrosis factor (TNF) inhibitors [12-14], abatacept [13-15] and the Janus kinase (JAK) inhibitor tofacitinib [16]. The MBDA score has not yet been assessed in patients treated with rituximab.

Rituximab is an anti-CD20 monoclonal antibody. CD20 is expressed by pre- $\mathrm{B}$ and mature $\mathrm{B}$ cells, which produce a number of pro-inflammatory cytokines, such as interleukin6 (IL-6) and TNF. By depleting CD20+ B cells, rituximab treatment leads to a decrease in these pro-inflammatory cytokines $[17,18]$, thereby reducing clinical disease activity. IL- 6 and TNF are 2 of the 12 biomarkers of the MBDA score. It is not known if the clinical response to rituximab is paralleled by changes in the biomarker profile of the MBDA score.

The purpose of the current study was to assess the ability of the MBDA score to measure disease activity upon and track response to treatment with rituximab and, if so, if this would be mainly explained by the objective component of the DAS28 (acute phase reactants). Furthermore, we investigated the ability of the MBDA score to predict radiographic progression and change in serum bone turnover markers upon rituximab treatment.

\section{Methods}

\section{Study population and treatment protocol}

We used data from three prospective cohort studies in which adult, refractory RA patients were treated with rituximab because of active disease despite conventional treatment (e.g. a combination of DMARDs, including maximum tolerable doses of a conventional synthetic (cs)DMARD and/or TNF inhibitor): one cohort from the Leiden University Medical Center (LUMC) [19] and one from the University Medical Center (UMC) Utrecht [20], both in the Netherlands, and the HORUS cohort in the United Kingdom [21]. All patients with available serum samples were selected from the cohorts. Patients received rituximab $1000 \mathrm{mg}$ intravenously on days 1 and 15, after an infusion with intravenous methylprednisolone $100 \mathrm{mg}$. Patients were followed for at least 1 year from baseline. For the current study, we used disease activity data from the first 6 months following rituximab infusion, to avoid potentially confounding effects from repeat rituximab infusions in some patients.

\section{Clinical assessments and serum samples}

Demographics, disease duration, smoking status (no or yes) and serum status for rheumatoid factor (RF) and for autoantibodies against citrullinated peptides (ACPA) were assessed at baseline. Swollen and tender joint counts assessing 28 joints (SJC28, TJC28), patient visual analogue scale (VAS) for general health $(\mathrm{GH})$, and health assessment questionnaire (HAQ) were obtained for patients at baseline and 6 months, as were erythrocyte sedimentation rate (ESR), CRP and high-sensitivity (hs)CRP (the latter only in HORUS). The DAS28 was calculated using both ESR and hsCRP. EULAR response at 6 months was determined using DAS28-ESR [22]. Radiographs of hands and feet were obtained at baseline and at 12 months (UMC Utrecht cohort) and radiographic progression was assessed using the Sharp/van der Heijde score (SHS) by one reader. Clinically important radiographic progression was defined as $\triangle \mathrm{SHS} \geq 5$ [23]. In the HORUS cohort, serum bone formation markers (BAP (bone-specific alkaline phosphatase), P1NP (procollagen 
type 1 amino-terminal propeptide), DKK1 (Dickkopf-1), sclerostin) and bone resorption markers (TRAP5b (tartrate-resistant acid phosphatase isoenzyme $5 \mathrm{~b}$ ), $\beta \mathrm{CTX}$ (beta-isomerised carboxy terminal telopeptide of type I collagen)) were determined at baseline and at 6 months (Additional file 1).

\section{Determination of the MBDA score}

Serum samples were collected at baseline in all three cohorts, and at 6 months in the UMC Utrecht and HORUS cohorts. Samples were shipped frozen to Crescendo Bioscience, Inc. (South San Francisco, CA, USA) for measurement of the 12 MBDA biomarkers. The biomarkers represent inflammatory and destructive processes: vascular cell adhesion molecule-1 (VCAM-1), epidermal growth factor (EGF), vascular endothelial growth factor A (VEGF-A), IL-6, TNF receptor type 1 (TNF-R1), matrix metalloproteinase 1 (MMP-1), MMP-3, human cartilage glycoprotein-39 (YKL-40), leptin, resistin, serum amyloid A (SAA) and CRP. The MBDA biomarkers were measured by electrochemiluminescence-based multiplexed sandwich immunoassays (Meso Scale Discovery, Rockville, MD, USA) using the same types of reagents and instrument and the same algorithm as described previously $[6,7]$.

\section{Statistical analyses}

Baseline characteristics were assessed using descriptive statistics. Differences between the three cohorts were analysed using one-way analysis of variance, Kruskal-Wallis test or chi-square test, as appropriate.

Spearman's rank correlations ( $r$ ) were analysed for values at baseline, at 6 months and for change from baseline to 6 months $(\Delta)$ between MBDA score and the following measures: DAS28-ESR, DAS28-hsCRP, ESR, CRP, hsCRP, SJC28, TJC28, VAS-GH, HAQ, SHS (UMC Utrecht cohort), bone turnover markers (HORUS cohort). Multivariable linear regression analyses were performed using these measures as dependent variable and the MBDA score as independent variable, with adjustment by age, gender,

Table 1 Patient characteristics at baseline

\begin{tabular}{|c|c|c|c|c|c|}
\hline & All, $n=57$ & HORUS, $n=26$ & UMC Utrecht, $n=20$ & LUMC, $n=11$ & $p$ value \\
\hline Female, n (\%) & $41(72)$ & $22(85)$ & $12(60)$ & $7(64)$ & $0.15^{1}$ \\
\hline Age in years, mean (SD) & $56.6(11.2)$ & $59.3(10.8)$ & $56.7(11.6)$ & $50.1(9.5)$ & $0.07^{2}$ \\
\hline Disease duration in years, median (IQR) & $11.5(6.3-16.4)$ & $9.9(4.1-14.4)$ & $13.4(8.4-17.6)$ & $13.0(5.2-15.5)$ & $0.46^{3}$ \\
\hline \multicolumn{6}{|l|}{ Smoking status, number (\%) } \\
\hline No & $37(65)$ & $16(62)$ & $12(60)$ & $9(82)$ & \multirow[t]{2}{*}{$0.42^{1}$} \\
\hline Yes & $20(35)$ & $10(38)$ & $8(40)$ & $2(18)$ & \\
\hline RF positive, number (\%) & $51(90)$ & $23(89)$ & $19(95)$ & $9(82)$ & $0.51^{1}$ \\
\hline ACPA positive, number (\%) & $44(80)$ & $19(79), n=24$ & $17(85)$ & $8(73)$ & $0.71^{1}$ \\
\hline \multicolumn{6}{|l|}{ Menopausal status, females (\%) } \\
\hline Pre-menopausal & $14(25)$ & $6(23)$ & $5(25)$ & $3(27)$ & \multirow[t]{2}{*}{$0.30^{1}$} \\
\hline Post-menopausal & $27(47)$ & $16(62)$ & $7(35)$ & $4(36)$ & \\
\hline SJC28, median (IQR) & $9(4-16)$ & $9(4-15)$ & $12(8-19), n=19$ & $4(1-10), n=8$ & $0.02^{3}$ \\
\hline TJC28, median (IQR) & $15(10-23)$ & $16(11-25)$ & $14(8-17), n=19$ & $13(5-24), n=8$ & $0.35^{3}$ \\
\hline VAS-GH, 0-100 mm (worst), median (IQR) & $64(45-73)$ & $69(40-78)$ & $57(46-69), n=19$ & $65(53-84), n=8$ & $0.36^{3}$ \\
\hline ESR, mm/h, median (IQR) & $37(21-51)$ & $32(12-41), n=24$ & $52(21-91), n=18$ & $32(29-44), n=7$ & $0.02^{3}$ \\
\hline CRP, mg/L, median (IQR) & $15(6-34)$ & $11(5-25), n=25$ & $29(11-50), n=18$ & $13(5-56), n=5$ & $0.02^{3}$ \\
\hline hsCRP, mg/L, median (IQR) & NA & $10(3-26)$ & NA & NA & NA \\
\hline DAS28-ESR, median (IQR) & $6.3(5.4-7.1)$ & $6.2(5.0-7.2), n=25$ & $6.6(5.8-7.1), n=18$ & $6.1(3.8-7.3), n=8$ & $0.64^{3}$ \\
\hline DAS28-hsCRP, median (IQR) & NA & $5.8(4.6-6.8)$ & NA & NA & NA \\
\hline MBDA score, median (IQR) & $54(44-70)$ & $51(44-67), n=25$ & $64(49-74)$ & $55(34-71), n=7$ & $0.15^{3}$ \\
\hline HAQ, median (IQR) & $1.8(1.4-2.1)$ & $1.9(1.7-2.1)$ & $1.5(1.1-1.9), n=11$ & $1.3(1.3-1.9), n=7$ & $0.02^{3}$ \\
\hline SHS, median (IQR) & $44(24-128)$ & NA & $61(29-142), n=19$ & $25(21-94), n=8$ & $0.34^{3}$ \\
\hline
\end{tabular}

$S D$ standard deviation, IQR interquartile range, $R F$ rheumatoid factor, ACPA anti-citrullinated protein antibodies, SJC28 swollen joint count assessing 28 joints, $T J C 28$ tender joint count assessing 28 joints, VAS-GH patient visual analogue scale for general health, ESR erythrocyte sedimentation rate, $m m / h$ millimetre/hour, $C R P C-$ reactive protein, $\mathrm{mg} / \mathrm{L}$ milligram/litre, $h s C R P$ high-sensitivity $C R P, D A S 28$ disease activity score assessing 28 joints, $M B D A$ multi-biomarker disease activity, $H A Q$ health assessment questionnaire, SHS Sharp/van der Heijde score, NA not applicable

${ }^{1}$ Differences between cohorts were analysed using chi-square test

${ }^{2}$ Differences between cohorts were analysed using one-way analysis of variance

${ }^{3}$ Differences between cohorts were analysed using Kruskal-Wallis test 
smoking status (no or yes), RF status, ACPA status, and cohort. Bone turnover markers were additionally adjusted for menopausal status (pre-menopausal or post-menopausal) [24]. Logistic regression analysis was performed to assess the association between baseline MBDA score or $\triangle \mathrm{MBDA}$ score and EULAR response (good or moderate) at 6 months, with adjustment by the same covariates.

Two-sided $p$ values $<0.05$ were considered statistically significant. All statistical analyses were performed using IBM SPSS Statistics 21 software (IBM Corp, Armonk, NY, USA).

\section{Results}

\section{Patient characteristics at baseline}

Baseline characteristics were generally typical of those for patients with established RA starting rituximab treatment and were mostly similar between the three cohorts. SJC28, ESR, CRP and HAQ were statistically significantly different between the three cohorts (Table 1). Overall, 90\% and $80 \%$ of patients were seropositive for RF or ACPA, respectively.

\section{MBDA score and DAS28 at baseline and 6 months}

At baseline the median MBDA score was 54 (interquartile range (IQR) $44-70, n=52$ ), with high $(>44)$, moderate (30-44) or low (<30; [7]) scores observed in 40 (77\%), 7 (13\%) and 5 (10\%) patients, respectively. At 6 months the median MBDA score was 51 (IQR 39-58, $n=42$ ), with high, moderate or low scores observed in 26 (62\%), 11 $(26 \%)$ and 5 patients (12\%), respectively. The median $\triangle$ MBDA score was -7 (IQR $-19-3, n=42$ ).

At baseline and at 6 months, the median values for DAS28-ESR were 6.3 (IQR 5.4-7.1, $n=51$ ) and 5.0 (IQR $4.2-6.2, n=45)$, respectively, and the median $\triangle \mathrm{DAS} 28$-ESR was -1.0 (IQR -2.0 to $-0.1, n=42$ ). At baseline and at 6 months, the median values for DAS28-hsCRP were 5.8 (IQR 4.6-6.8, $n=26$ ) and 4.7 (IQR 3.8-6.2, $n=26$ ), respectively, and the median $\triangle \mathrm{DAS} 28$-hsCRP was -0.9 (IQR $-1.6-0.1, n=26$ ).

\section{Correlation between MBDA score and disease activity measures}

Correlations between MBDA score and DAS28 and their changes over time are shown in Fig. 1. A significant Spearman's correlation was found between MBDA score and DAS28-ESR at baseline $(r=0.52, p<0.01)$ and at 6 months $(r=0.49, p<0.01)$. $\triangle$ MBDA score from baseline to 6 months was significantly correlated with $\triangle$ DAS28-ESR $(r=0.60, p<0.01)$.

Similarly, the MBDA score was significantly correlated with DAS28-hsCRP at baseline $(r=0.51, p<0.01)$ and at 6 months $(r=0.45, p=0.03)$. $\triangle$ MBDA score from baseline to 6 months was significantly correlated with $\triangle \mathrm{DAS} 28$ $\operatorname{hsCRP}(r=0.48, p=0.02)$.
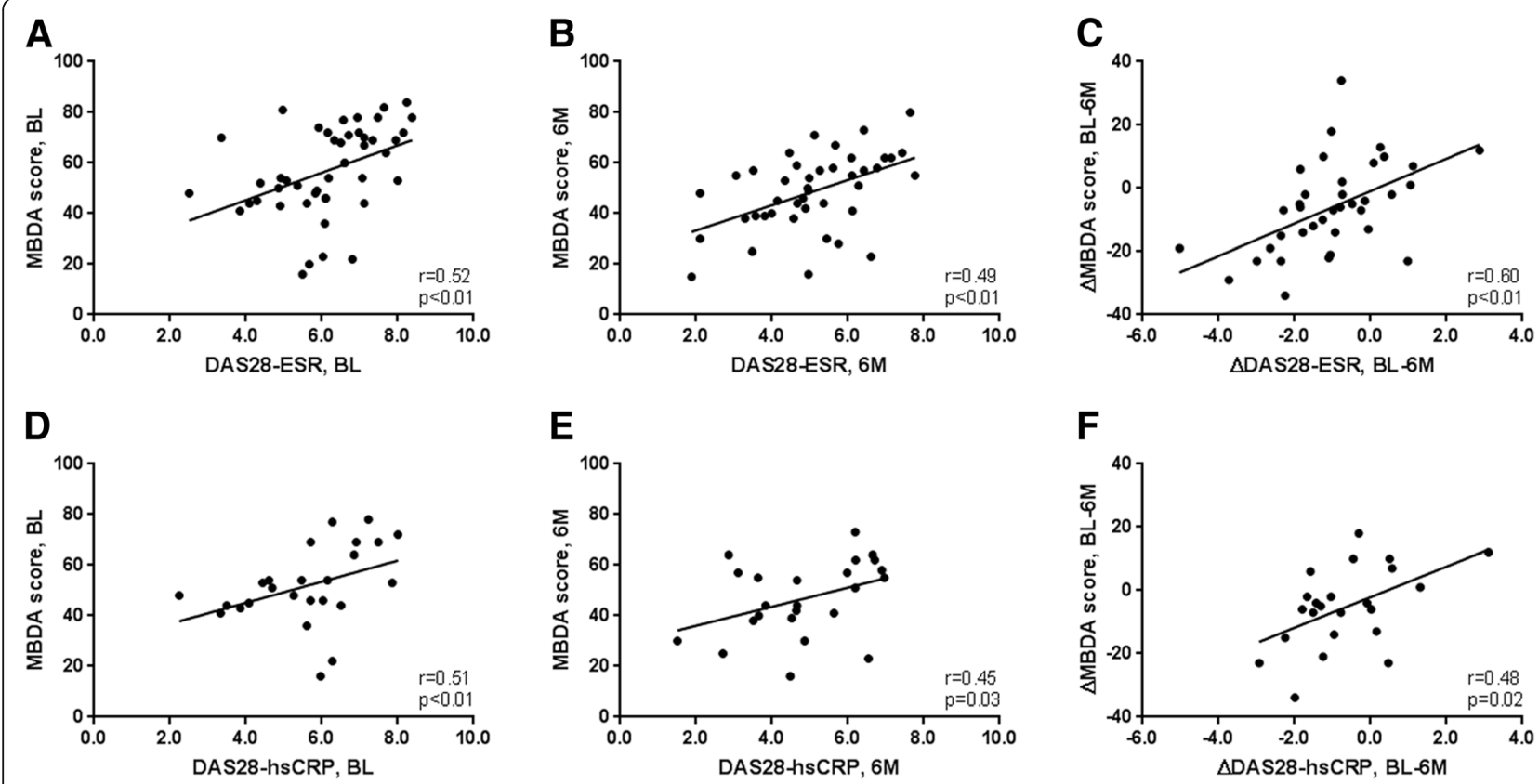

Fig. 1 Correlation between MBDA score and DAS28. a MBDA score versus DAS28-ESR at baseline $(n=46)$. b MBDA score versus DAS28-ESR at 6 months $(n=42)$. c $\triangle M B D A$ score versus $\triangle D A S 28$-ESR, from baseline to 6 months $(n=38)$. $\mathbf{d}$ MBDA score versus DAS28-hsCRP at baseline $(n=25)$. e MBDA score versus DAS28-hsCRP at 6 months $(n=24)$. f $\triangle$ MBDA score versus $\triangle D A S 28$-hsCRP, from baseline to 6 months $(n=23)$. Negative change values represent improvement over 6 months 
MBDA score was significantly correlated with ESR, hsCRP and CRP, as was also true for their changes from baseline to 6 months (Table 2).

Correlations were not significant between the MBDA score and SJC28, TJC28, VAS-GH or HAQ, except for $\triangle$ SJC28 and $\triangle$ VAS-GH from baseline to 6 months (Table 2).

The results of the multivariable regression analysis resembled those of the correlation analyses, except that the associations between $\triangle \mathrm{MBDA}$ score versus $\triangle \mathrm{ESR}$ and $\triangle$ SJC28 were not statistically significant and the association between MBDA score versus TJC28 at baseline was statistically significant (Table 2).

\section{Association between MBDA score and EULAR response}

At 6 months, 21 patients (48\%) were classified as non-, 19 patients (43\%) as moderate and 4 patients (9\%) as good EULAR responders. The distribution of values for $\triangle \mathrm{MBDA}$ score within each EULAR response category is shown in Fig. 2. $\triangle$ MBDA score from baseline to 6 months was significantly associated with EULAR response (good or moderate) versus non-response at 6 months (odds ratio (OR): 0.93 (95\% CI $=0.88-0.98, p=0.01$ ) per unit change in MBDA score, Fig. 2). Adjusted by age, gender, smoking status, RF status, ACPA status, and cohort, this association remained statistically significant (OR: $0.89(95 \% \mathrm{CI}=0.81-$ $0.98, p=0.02)$ per unit change in MBDA score).

Table 2 Correlations and associations between the MBDA score and disease activity measures

\begin{tabular}{|c|c|c|c|c|c|c|}
\hline Measure & $\begin{array}{l}\text { Time point or period for } \\
\text { comparison with MBDA score }\end{array}$ & Number of available samples & r & $p$ value & $\beta(95 \% \mathrm{Cl})^{1}$ & $p$ value \\
\hline \multirow[t]{3}{*}{ DAS28-ESR } & $\mathrm{BL}$ & 46 & 0.52 & $<0.01$ & $0.05(0.02-0.07)$ & $<0.01$ \\
\hline & $6 \mathrm{M}$ & 42 & 0.49 & $<0.01$ & $0.06(0.02-0.09)$ & 0.01 \\
\hline & $\Delta$ & 38 & 0.60 & $<0.01$ & $0.05(0.01-0.08)$ & 0.02 \\
\hline \multirow[t]{3}{*}{ ESR } & $\mathrm{BL}$ & 44 & 0.75 & $<0.01$ & $1.20(0.71-1.70)$ & $<0.01$ \\
\hline & $6 \mathrm{M}$ & 42 & 0.66 & $<0.01$ & $0.81(0.36-1.26)$ & $<0.01$ \\
\hline & $\Delta$ & 37 & 0.48 & $<0.01$ & $0.57(-0.03-1.17)$ & 0.06 \\
\hline \multirow[t]{3}{*}{ DAS28-hsCRP² } & $B L$ & 25 & 0.51 & $<0.01$ & $0.06(0.02-0.10)$ & 0.01 \\
\hline & $6 \mathrm{M}$ & 24 & 0.45 & 0.03 & $0.06(0.02-0.10)$ & $<0.01$ \\
\hline & $\triangle$ & 23 & 0.48 & 0.02 & $0.05(0.00-0.09)$ & $<0.05$ \\
\hline \multirow[t]{3}{*}{$\mathrm{hsCRP}{ }^{2}$} & $B L$ & 25 & 0.80 & $<0.01$ & $1.24(0.72-1.76)$ & $<0.01$ \\
\hline & $6 \mathrm{M}$ & 24 & 0.80 & $<0.01$ & $0.75(0.41-1.10)$ & $<0.01$ \\
\hline & $\Delta$ & 23 & 0.71 & $<0.01$ & $0.90(0.60-1.21)$ & $<0.01$ \\
\hline \multirow[t]{3}{*}{ CRP } & $B L$ & 46 & 0.75 & $<0.01$ & $1.07(0.62-1.52)$ & $<0.01$ \\
\hline & $6 \mathrm{M}$ & 40 & 0.76 & $<0.01$ & $0.82(0.58-1.06)$ & $<0.01$ \\
\hline & $\Delta$ & 37 & 0.59 & $<0.01$ & $0.68(0.18-1.19)$ & $<0.01$ \\
\hline \multirow[t]{3}{*}{ SJC28 } & $B L$ & 48 & 0.15 & 0.32 & $0.10(-0.06-0.26)$ & 0.22 \\
\hline & $6 \mathrm{M}$ & 42 & 0.26 & 0.10 & $0.14(-0.01-0.28)$ & 0.06 \\
\hline & $\Delta$ & 40 & 0.42 & $<0.01$ & $0.12(-0.04-0.29)$ & 0.14 \\
\hline \multirow[t]{3}{*}{ TJC28 } & $B L$ & 48 & 0.23 & 0.12 & $0.17(0.02-0.32)$ & 0.03 \\
\hline & $6 \mathrm{M}$ & 42 & 0.25 & 0.11 & $0.17(-0.01-0.34)$ & 0.06 \\
\hline & $\Delta$ & 40 & 0.28 & 0.08 & $0.04(-0.15-0.23)$ & 0.67 \\
\hline \multirow[t]{3}{*}{ VAS-GH } & $B L$ & 48 & 0.20 & 0.18 & $0.34(-0.12-0.79)$ & 0.14 \\
\hline & $6 \mathrm{M}$ & 42 & 0.27 & 0.09 & $0.46(-0.08-0.99)$ & 0.09 \\
\hline & $\Delta$ & 40 & 0.36 & 0.02 & $0.74(0.08-1.40)$ & 0.03 \\
\hline \multirow[t]{3}{*}{ HAQ } & $B L$ & 39 & 0.02 & 0.91 & $0.06(-0.06-0.02)$ & 0.30 \\
\hline & $6 \mathrm{M}$ & 41 & -0.03 & 0.85 & $-0.01(-0.01-0.01)$ & 0.84 \\
\hline & $\Delta$ & 34 & 0.19 & 0.28 & $0.00(-0.01-0.01)$ & 0.77 \\
\hline
\end{tabular}

DAS28 disease activity score using 28 joints, ESR erythrocyte sedimentation rate, $h s C R P$ high-sensitivity C-reactive protein, SJC28 swollen joint count assessing 28 joints, TJC28 tender joint count assessing 28 joints, VAS-GH patient visual analogue scale for general health, HAQ health assessment questionnaire, MBDA multibiomarker disease activity, $B L$ MBDA score and measure both at baseline, $6 \mathrm{M}$ MBDA score and measure both at month $6, \triangle$ change in MBDA score and measure, both from baseline to month $6, r$ Spearman's rank correlation, $C l$ confidence interval

${ }^{1} \beta$ : regression coefficient from multivariable linear regression analysis, after adjustment by age, gender, smoking status, RF status, ACPA status, and cohort ${ }^{2}$ HORUS cohort only 


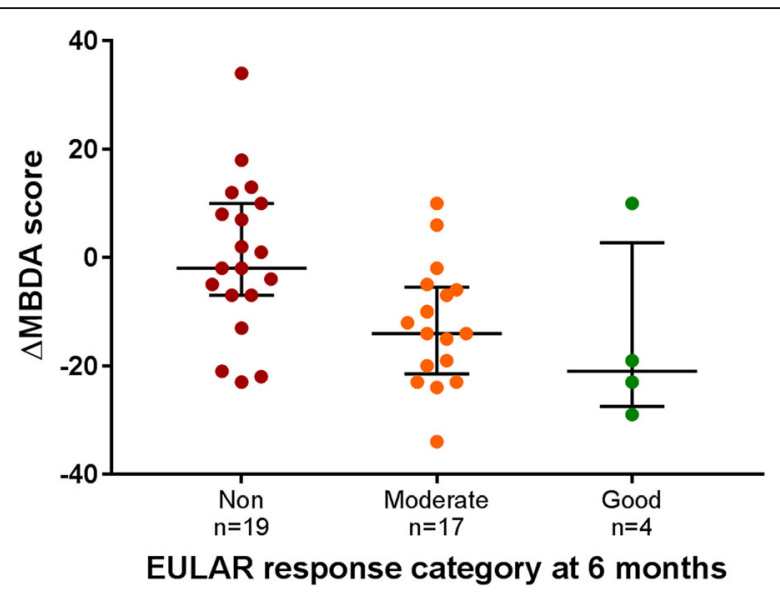

Fig. 2 Change in MBDA score by EULAR response category at 6 months. Individual patient values of $\triangle M B D A$ score from baseline to 6 months are shown as dots, grouped by EULAR response category at 6 months (EULAR non-, moderate, good response). Dark lines represent median values. Whiskers represent interquartile ranges (25th-75th). $\Delta$ : change

The MBDA score at baseline was not associated with EULAR response (good or moderate) versus non-response at 6 months, with OR of $1.01(95 \% \mathrm{CI}=0.98-1.05, p=0.35)$ per unit MBDA score, even after adjustment by age, gender, smoking status, RF status, ACPA status, and cohort (OR: $1.03(95 \% \mathrm{CI}=0.98-1.08, p=0.27)$ per unit MBDA score) .

\section{Correlation between MBDA score and radiographic progression or bone turnover markers}

For the 11 patients with radiographs available at baseline and 12 months, all from the UMC Utrecht cohort, the median $\triangle$ SHS was 3 (IQR -1-12). At baseline, low, moderate and high MBDA scores were observed in 1, 1 and 9 patients, respectively. Radiographic progression $(\triangle \mathrm{SHS} \geq$ 5 ) in patients with low, moderate and high MBDA scores was observed in $0(0 \%), 0(0 \%)$ and $5(56 \%)$ patients, respectively.

No significant Spearman's correlation was found between MBDA score or $\triangle \mathrm{MBDA}$ score and $\triangle \mathrm{SHS}$ over 12 months, nor bone turnover markers (Table 3). Similar findings were obtained with multivariable regression analysis adjusted by age, gender (menopausal status for bone turnover markers), smoking status, RF status and ACPA status (Table 3).

\section{Discussion}

The MBDA score has been shown to track response to a variety of DMARDs. We found significant correlations between the MBDA score and DAS28-ESR as well as DAS28-hsCRP at baseline and at 6 months, and between $\triangle M B D A$ score and $\triangle \mathrm{DAS} 28-\mathrm{ESR}$ and $\triangle \mathrm{DAS} 28$-hsCRP from baseline to 6 months in patients treated with rituximab. Moreover, $\triangle \mathrm{MBDA}$ score was significantly associated with EULAR response to rituximab treatment. This is the first time it has been shown that the MBDA score can be used to track disease activity in RA patients upon treatment with rituximab and that change in the MBDA score reflects response to rituximab treatment.

Our findings on the MBDA score are consistent with several previous studies in RA patients upon treatment with other cs-, biological, or targeted synthetic DMARDs $[6-8,10-16]$.

In our study, we additionally investigated if the MBDA score correlated with the individual components of the DAS28. We found correlations between the MBDA score or $\triangle \mathrm{MBDA}$ score and ESR, hsCRP or their changes, but found limited correlations between the MBDA score and the other DAS28 components. The correlation between the MBDA score and the DAS28 thus seems predominantly dependent on the biochemical components of the DAS28, the ESR or (hs)CRP. It would be of interest to assess the additional value of the MBDA score above ESR or CRP alone, but the present study was not powered to analyse this. A larger study has reported that an increase in TJC, SJC and patient global assessment was paralleled by an increase in MBDA score; and that, in patients positive for either RF and/or ACPA, an MBDA score excluding CRP was a significant predictor of both DAS28-CRP, and of DAS28 without any CRP or ESR component [7].

In addition, MBDA score appeared to be more sensitive for detecting inflammation than ESR or CRP. A study of 9135 RA patients with active disease found that ESR and CRP were normal in the majority [25]. In other studies, MBDA score was often elevated in such patients [26] and, when it was, risk of radiographic progression was increased $[9,27]$. In patients with disproportionally high subjective disease activity components (e.g. high tender joint counts with low ESR or CRP) the MBDA score might be an important alternative disease activity measure. We could not address this hypothesis, as no patients with normal ESR or normal CRP (defined as $\leq 1 \mathrm{~mm} / \mathrm{h}$ or $\mathrm{mg} / \mathrm{L}$ ) were included in this study.

Previous studies have shown that the MBDA score was a significant predictor of radiographic progression, both in early and established RA [9, 27-30]. In the present study, all patients with clinically important radiographic progression $(\triangle \mathrm{SHS} \geq 5)$ had a high MBDA score at baseline. This result resembles the findings in previous studies $[9,27-30]$. We did not find a significant Spearman's correlation between baseline MBDA score and $\triangle \mathrm{SHS}$ in patients treated with rituximab, possibly due to the small number of patients $(n=11)$ and the limited observation period.

B-cell depletion upon rituximab treatment has been shown to be most effective in RF-positive patients [31], and has been suggested to be associated with ACPA positivity [32]. In future studies, it would be of interest to stratify the performance of the MBDA score in rituximab-treated RA patients according to RF and ACPA status. 
Table 3 Correlations and associations between the MBDA score and radiographic progression or bone turnover markers

\begin{tabular}{|c|c|c|c|c|c|c|}
\hline Measure & $\begin{array}{l}\text { Time point or period } \\
\text { for comparison with } \\
\text { MBDA score }\end{array}$ & Number of available samples & r & $p$ value & $\beta(95 \% \mathrm{Cl})^{1}$ & $p$ value \\
\hline \multirow[t]{3}{*}{$\overline{\Delta S H S}$ (baseline-12 months) ${ }^{2}$} & $B L$ & 11 & 0.19 & 0.57 & $0.34(-0.33-1.01)$ & 0.23 \\
\hline & $6 \mathrm{M}$ & 11 & 0.18 & 0.60 & $0.18(-0.72-1.08)$ & 0.62 \\
\hline & $\Delta$ & 11 & 0.19 & 0.57 & $-0.38(-1.35-0.60)$ & 0.34 \\
\hline \multicolumn{7}{|l|}{ Bone turnover markers ${ }^{3}$} \\
\hline$\beta C T X$ & $\Delta$ & 23 & 0.22 & 0.31 & $1.10(-6.79-8.99)$ & 0.77 \\
\hline P1NP & $\Delta$ & 23 & -0.14 & 0.54 & $-0.31(-2.05-1.42)$ & 0.70 \\
\hline BAP & $\Delta$ & 23 & -0.01 & 0.98 & $-0.01(-0.17-0.15)$ & 0.88 \\
\hline TRAP5b & $\Delta$ & 23 & -0.20 & 0.37 & $0.00(-0.03-0.03)$ & 0.99 \\
\hline DKK1 & $\Delta$ & 23 & 0.27 & 0.22 & $0.15(-0.40-0.69)$ & 0.57 \\
\hline Sclerostin & $\Delta$ & 23 & 0.11 & 0.63 & $0.10(-0.37-0.56)$ & 0.66 \\
\hline
\end{tabular}

SHS Sharp/van der Heijde score, $\beta C T X$ beta-isomerised carboxy terminal telopeptide of type I collagen, P1NP procollagen type 1 amino-terminal propeptide, TRAP5 $b$ tartrate-resistant acid phosphatase isoenzyme 5b, DKK1 Dickkopf-1, BAP bone-specific alkaline phosphatase, MBDA multi-biomarker disease activity, $B L$ MBDA score at baseline, $6 \mathrm{M}$ MBDA score at month $6, \triangle$ change (for SHS: $\triangle \mathrm{MBDA}$ score from baseline to month 6 and $\triangle \mathrm{SHS}$ from baseline to month 12; for bone turnover markers: both $\Delta$ from baseline to month 6), $r$ Spearman's rank correlation, $\mathrm{Cl}$ confidence interval

${ }^{1} \beta$ : regression coefficient from multivariable linear regression analysis, after adjustment by age, gender and/or menopausal status, smoking status, RF status, and ACPA status

${ }^{2}$ UMC Utrecht cohort only

${ }^{3}$ HORUS cohort only

Other studies have shown that rituximab treatment increases bone formation and decreases bone resorption in RA patients [33, 34]. For example, a significant correlation was found between the changes of DAS28 and $\beta C T X$ [34], showing that the anti-inflammatory therapeutic response with rituximab and the anti-resorptive effect on bone might be related. In future, in larger studies with longer follow-up, it may be of interest to investigate the relationship between the MBDA score and bone turnover.

\section{Conclusions}

In conclusion, we have shown, for the first time, that the MBDA score correlated with DAS28 following treatment with the B-cell depleting agent rituximab and that $\triangle M B D A$ score reflected the treatment response. Our findings are consistent with previous research in RA patients treated with other DMARDs.

\section{Additional file}

Additional file 1: Determination of bone turnover markers. (PDF $102 \mathrm{~kb}$ )

\section{Abbreviations}

ACPA: Autoantibodies against citrullinated peptides; ACR: American College of Rheumatology; BAP: Bone-specific alkaline phosphatase; Cl: Confidence interval; CRP: C-reactive protein; DAS28: Disease activity score assessing 28 joints; DKK1: Dickkopf-1; DMARDs: Disease modifying anti-rheumatic drugs; EGF: Epidermal growth factor; ESR: Erythrocyte sedimentation rate; EULAR: European League Against Rheumatism; HAQ: Health assessment questionnaire; hsCRP: High-sensitivity C-reactive protein; IL-6: Interleukin 6; JAK: Janus kinase; MBDA: Multi-biomarker disease activity; MMP-1: Matrix metalloproteinase 1; MMP-3: Matrix metalloproteinase 3; OR: Odds ratio;
P1NP: Procollagen type 1 amino-terminal propeptide; RA: Rheumatoid arthritis; RF: Rheumatoid factor; SAA: Serum amyloid A; SD: Standard deviation; SHS: Sharp/van der Heijde score; SJC28: Swollen joint count assessing 28 joints; TJC28: Tender joint count assessing 28 joints; TNF: Tumour necrosis factor; TNF-R1: TNF receptor type 1; TRAP5b: Tartrate-resistant acid phosphatase isoenzyme 5b; VAS-GH: Visual analogue scale measuring general health; VCAM-1: Vascular cell adhesion molecule-1; VEGF-A: Vascular endothelial growth factor A; YKL-40: Human cartilage glycoprotein-39; $\beta C T X$ : Betaisomerised carboxy terminal telopeptide of type I collagen

\section{Acknowledgements}

Not applicable.

Funding

Not applicable.

\section{Availability of data and materials}

The datasets used and/or analysed during the current study are available from the corresponding author on reasonable request.

\section{Authors' contributions}

NMTR contributed to the data analysis, interpretation of data and manuscript preparation. MJHdH contributed to the design of the study, data analysis, interpretation of data and manuscript preparation. GW, ME, JT and YKOT contributed to the acquisition of data, interpretation of data and manuscript preparation. FPJGL contributed to interpretation of data and manuscript preparation. $\mathrm{CCH}, \mathrm{XL}$ and EHS contributed to the design of the study, data analysis, interpretation of data and manuscript preparation. JMvL contributed to the design of the study, interpretation of data and manuscript preparation. All authors read and approved the final manuscript.

\section{Ethics approval and consent to participate}

All of the studies were approved by institutional review board and independent ethics committees at participating sites and were conducted in accordance with the Declaration of Helsinki (registration numbers: United Kingdom ISRCTN82317088, LUMC METC P05.037, UMC Utrecht METC 05/169). Because this was a post hoc analysis, no specific consent was obtained above that received for each individual trial. 


\section{Consent for publication}

Not applicable.

\section{Competing interests}

YKOT is supported by the Dutch Kidney Foundation (KJPB12.028 and 170KG04), Clinical Fellowship from the Netherlands Organization for Scientific Research (90713460). CCH and XL are employees of Crescendo Bioscience. EHS is an employee of Crescendo Bioscience and holds stock shares in Myriad Genetics. JMvL received fees from Arthrogene, MSD, Pfizer, Eli Lilly, and BMS and research grants from Astra Zeneca, Roche-Genentech. The other authors declare that they have no competing interests.

\section{Publisher's Note}

Springer Nature remains neutral with regard to jurisdictional claims in published maps and institutional affiliations.

\section{Author details}

'Department of Rheumatology \& Clinical Immunology, University Medical Center Utrecht, Heidelberglaan 100, 3508 GA Utrecht, The Netherlands. ${ }^{2}$ Department of Biochemistry, The James Cook University Hospital, Marton Road, Middlesborough TS4 3BW, UK. ${ }^{3}$ Department of Rheumatology and Rehabilitation, Suez Canal University, Suez Canal University Circular Road, Ismailia 411522, Egypt. ${ }^{4}$ Department of Nephrology, Leiden University Medical Center, Albinusdreef 2, 2333 ZA Leiden, The Netherlands. ${ }^{5}$ Crescendo Bioscience, 341 Oyster Point Blvd, South San Franscisco, CA 94080, USA

\section{Received: 14 August 2018 Accepted: 18 October 2018}

\section{Published online: 20 November 2018}

\section{References}

1. Smolen JS, Aletaha D, McInnes IB. Rheumatoid arthritis. Lancet. 2016;388: 2023-38.

2. Smolen JS, Landewé R, Bijlsma J, Burmester G, Chatzidionysiou K, Dougados $M$, et al. EULAR recommendations for the management of rheumatoid arthritis with synthetic and biological disease-modifying antirheumatic drugs: 2016 update. Ann Rheum Dis. 2017;76:960-77.

3. Singh JA, Saag KG, Bridges SL, AkI EA, Bannuru RR, Sullivan MC, et al. American College of Rheumatology guideline for the treatment of rheumatoid arthritis. Arthritis Rheumatol. 2016. 2015;68:1-26.

4. Gaujoux-Viala C, Mouterde G, Baillet A, Claudepierre P, Fautrel B, Le Loët X, et al. Evaluating disease activity in rheumatoid arthritis: Which composite index is best? A systematic literature analysis of studies comparing the psychometric properties of the DAS, DAS28, SDAI and CDAI. Joint Bone Spine. 2012;79:149-55.

5. Jacobs JWG, Ten Cate DF, van Laar JM. Monitoring of rheumatoid arthritis disease activity in individual patients: still a hurdle when implementing the treat-to-target principle in daily clinical practice. Rheumatology. 2015;54: 959-61.

6. Centola M, Cavet G, Shen Y, Ramanujan S, Knowlton N, Swan KA, et al. Development of a Multi-Biomarker Disease Activity Test for Rheumatoid Arthritis. PLoS One. 2013;8:e60635.

7. Curtis JR, Van Der Helm-Van Mil AH, Knevel R, Huizinga TW, Haney DJ, Shen $Y$, et al. Validation of a novel multibiomarker test to assess rheumatoid arthritis disease activity. Arthritis Care Res. 2012;64:1794-803.

8. Hirata S, Dirven L, Shen Y, Centola M, Cavet G, Lems WF, et al. A multibiomarker score measures rheumatoid arthritis disease activity in the best study. Rheumatology. 2013;52:1202-7.

9. Li W, Sasso EH, van der Helm-van Mil AHM, Huizinga TWJ. Relationship of multi-biomarker disease activity score and other risk factors with radiographic progression in an observational study of patients with rheumatoid arthritis. Rheumatology. 2016;55:357-66.

10. Bakker MF, Cavet G, Jacobs JW, Bijlsma JWJ, Haney DJ, Shen Y, et al. Performance of a multi-biomarker score measuring rheumatoid arthritis disease activity in the CAMERA tight control study. Ann Rheum Dis. 2012;71:1692-7.

11. Jurgens MS, Jacobs JW, Bijlsma JW, Bakker MF, Welsing PMJ, Tekstra J. The multi-biomarker disease activity test for assessing response to treatment strategies using methotrexate with or without prednisone in the CAMERA trial-II; 2014. p. 81-98.
12. Hirata S, Li W, Defranoux N, Cavet G, Bolce R, Yamaoka K, et al. A multibiomarker disease activity score tracks clinical response consistently in patients with rheumatoid arthritis treated with different anti-tumor necrosis factor therapies: A retrospective observational study. Mod Rheumatol. 2015;25:344-9.

13. Fleischmann R, Connolly SE, Maldonado MA, Schiff M. Brief report: estimating disease activity using multi-biomarker disease activity scores in rheumatoid arthritis patients treated with abatacept or adalimumab. Arthritis Rheumatol. 2016:68:2083-9.

14. Curtis JR, Wright GC, Strand V, Davis CS, Hitraya E, Sasso EH. Reanalysis of the multi-biomarker disease activity score for assessing disease activity in the abatacept versus adalimumab comparison in biologic-naive rheumatoid arthritis subjects with background methotrexate study: Comment on the article by Fleischmann. Arthritis Rheumatol. 2017;69:863-5.

15. Haney D, Cavet G, Durez P, Alten R, Burmester G, Tak PP, et al. Correlation of a multi-biomarker disease activity response assessment to disease activity score 28 (C-reactive protein) response assessment and Omeract Ramris scores in a placebo-controlled rheumatoid arthritis clinical trial with abatacept (ASSET). ACR. 2012. Abstract 2159.

16. Yamaoka K, Kubo S, Li W, Sonomoto K, Hirata S, Sasso EH, et al. FRI0333 effects of tofacitinib treatment on leptin and other components of the multi-biomarker disease activity score in patients with rheumatoid arthritis. Ann Rheum Dis. 2014;73:507-8.

17. Shaw T, Quan J, Totoritis MC. B cell therapy for rheumatoid arthritis: the rituximab (anti-CD20) experience. Ann Rheum Dis. 2003;62(Suppl 2):ii55-9.

18. Mok CC. Rituximab for the treatment of rheumatoid arthritis: an update. Drug Des Devel Ther. 2013;8:87.

19. Teng YO, Wheater G, Hogan VE, Stocks P, Levarht EN, Huizinga TWJ, et al. Induction of long-term B-cell depletion in refractory rheumatoid arthritis patients preferentially affects autoreactive more than protective humoral immunity. Arthritis Res Ther. 2012;14:57.

20. Teng YKO, Tekstra J, Breedveld FC, Lafeber F, Bijlsma JWJ, Van Laar JM Rituximab fixed retreatment versus on-demand retreatment in refractory rheumatoid arthritis: comparison of two B cell depleting treatment strategies. Ann Rheum Dis. 2009;68:1075-7.

21. Elshahaly M, Wheater G, Naraghi K, Tuck SP, Datta HK, Ng W-F, et al. Changes in bone density and bone turnover in patients with rheumatoid arthritis treated with rituximab, a B cell depleting monoclonal antibody (HORUS TRIAL). BMC Musculoskelet Disord. 2013;14:A10.

22. van Riel PL, van Gestel $A M$, van de Putte $L B$. Development and validation of response criteria in rheumatoid arthritis: steps towards an international consensus on prognostic markers. Br J Rheumatol. 1996;35(Suppl 2):4-7.

23. Bruynesteyn K, Der HV, Boers M, Saudan A, Peloso P, Paulus H, et al. Determination of the minimal clinically important difference in rheumatoid arthritis joint damage of the Sharp/van der Heijde and Larsen/ Scott scoring methods by clinical experts and comparison with the smallest detectable difference. Arthritis Rheum. 2002;46:913-20.

24. Curtis JR, Greenberg JD, Harrold LR, Kremer JM, Palmer JL. Influence of obesity, age, and comorbidities on the multi-biomarker disease activity test in rheumatoid arthritis. Semin Arthritis Rheum. 2018;47:472-7.

25. Kay J, Morgacheva O, Messing SP, Kremer JM, Greenberg JD, Reed GW, et al. Clinical disease activity and acute phase reactant levels are discordant among patients with active rheumatoid arthritis: acute phase reactant levels contribute separately to predicting outcome at one year. Arthritis Res Ther. 2014;16:R40.

26. Lee YC, Hackett J, Frits M, lannaccone CK, Shadick NA, Weinblatt ME, et al. Multibiomarker disease activity score and C-reactive protein in a crosssectional observational study of patients with rheumatoid arthritis with and without concomitant fibromyalgia. Rheumatology. 2016;55:640-8.

27. van der Helm-van Mil AHM, Knevel R, Cavet G, Huizinga TWJ, Haney DJ. An evaluation of molecular and clinical remission in rheumatoid arthritis by assessing radiographic progression. Rheumatology. 2013;52:839-46.

28. Markusse IM, Dirven L, Van Den Broek M, Bijkerk C, Han KH, Ronday HK, et al. A multibiomarker disease activity score for rheumatoid arthritis predicts radiographic joint damage in the best study. J Rheumatol. 2014;41:2114-9.

29. Hirata S, Li W, Kubo S, Fukuyo S, Mizuno Y, Hanami K, et al. Association of the multi-biomarker disease activity score with joint destruction in patients with rheumatoid arthritis receiving tumor necrosis factor-alpha inhibitor treatment in clinical practice. Mod Rheumatol. 2016;26:850-6.

30. Hambardzumyan K, Bolce R, Saevarsdottir S, Cruickshank SE, Sasso EH, Chernoff $\mathrm{D}$, et al. Pretreatment multi-biomarker disease activity score and 
radiographic progression in early RA: Results from the SWEFOT trial. Ann Rheum Dis. 2015;74:1102-9.

31. Benucci M, Manfredi M, Sarzi-Puttini P, Atzeni F. Predictive factors of response to rituximab therapy in rheumatoid arthritis: What do we know today? Autoimmun Rev. 2010;9:801-3.

32. Cuppen BVJ, Welsing PMJ, Sprengers JJ, Bijlsma JWJ, Marijnissen ACA, van Laar JM, et al. Personalized biological treatment for rheumatoid arthritis: a systematic review with a focus on clinical applicability. Rheumatology. 2016:55:826-39.

33. Zerbini CAF, Clark P, Mendez-Sanchez L, Pereira RMR, Messina OD, Uña CR et al. Biologic therapies and bone loss in rheumatoid arthritis. Osteoporos Int. 2017;28:429-46.

34. Wheater G, Hogan VE, Teng YKO, Tekstra J, Lafeber FP, Huizinga TWJ, et al. Suppression of bone turnover by B-cell depletion in patients with rheumatoid arthritis. Osteoporos Int. 2011;22:3067-72.

Ready to submit your research? Choose BMC and benefit from:

- fast, convenient online submission

- thorough peer review by experienced researchers in your field

- rapid publication on acceptance

- support for research data, including large and complex data types

- gold Open Access which fosters wider collaboration and increased citations

- maximum visibility for your research: over $100 \mathrm{M}$ website views per year

At BMC, research is always in progress.

Learn more biomedcentral.com/submissions 